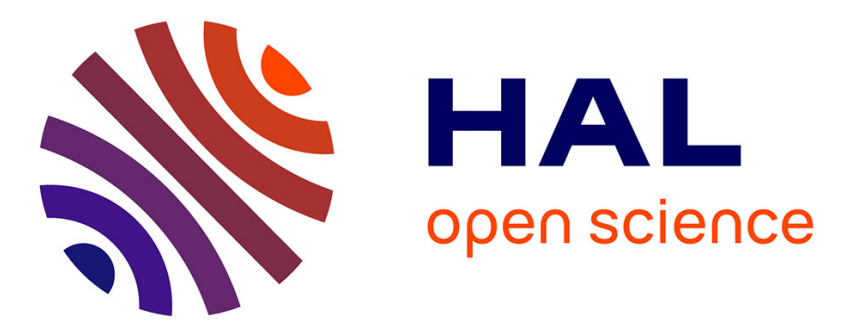

\title{
Analyse des relations de compétition dans une association de luzerne (Medicago sativa L.) et de dactyle (Dactylis glomerata L.). II. Effets sur la nutrition azotée des deux espèces
}

Pablo Cruz, Gilles Lemaire

\section{To cite this version:}

Pablo Cruz, Gilles Lemaire. Analyse des relations de compétition dans une association de luzerne (Medicago sativa L.) et de dactyle (Dactylis glomerata L.). II. Effets sur la nutrition azotée des deux espèces. Agronomie, 1986, 6 (8), pp.735-742. hal-00884931

\section{HAL Id: hal-00884931 https://hal.science/hal-00884931}

Submitted on 1 Jan 1986

HAL is a multi-disciplinary open access archive for the deposit and dissemination of scientific research documents, whether they are published or not. The documents may come from teaching and research institutions in France or abroad, or from public or private research centers.
L'archive ouverte pluridisciplinaire HAL, est destinée au dépôt et à la diffusion de documents scientifiques de niveau recherche, publiés ou non, émanant des établissements d'enseignement et de recherche français ou étrangers, des laboratoires publics ou privés. 


\title{
Analyse des relations de compétition dans une association de luzerne (Medicago sativa L.) et de dactyle (Dactylis glomerata L.). II. Effets sur la nutrition azotée des deux espèces
}

\author{
Pablo CRUZ \& Gilles LEMAIRE
}

I.N.R.A., Laboratoire d'Agronomie de la Prairie, F 86600 Lusignan

RÉSUMÉ

\begin{abstract}
Nous avons étudié, au sein d'une association luzerne-dactyle, les modifications du niveau de nutrition azotée des 2 espèces en réponse à la présence du partenaire et à l'augmentation du niveau de disponibilité en azote dans le sol. Une méthode indirecte, qui relie les teneurs en azote au degré de croissance du couvert végétal, nous a permis de suivre ces modifications tout au long des différentes repousses.

L'effet bénéfique de la présence de la luzerne sur la nutrition azotée du dactyle associé n'a pas pu être mis en évidence. Au contraire, la légumineuse peut devenir très compétitive pour cet élément nutritif.

Tout comme il a été constaté dans l'analyse de la croissance en matière sèche des partenaires dans l'association, c'est l'espèce qui a les vitesses de croissance les plus rapides à un moment donné, qui utilise le mieux l'azote minéral du sol.

Tout apport d'azote sur la culture associée amplifie la dominance d'un partenaire sur l'autre. Les conséquences sur l'utilisation des associations légumineuses-graminées sont discutées.

Dans une culture pure de luzerne, il semblerait que la forte compétition intraspécifique puisse avoir un effet dépressif sur la capacité de ficiation de l'azote atmosphérique par cette espèce.
\end{abstract}

Mots clés additionnels : Courbes de dilution, teneurs en azote, Medicago sativa, Dactylis glomerata, compétition.

Analysis of competition in a lucerne-cocksfoot association. II. Effects on the nitrogen nutrition of the 2 species.

The modification of nitrogen nutrition of lucerne and cocksfoot in association compared with pure stands was studied with different levels of nitrogen application. A relationship between nitrogen content and dry matter accumulation in aerial parts during regrowth Np.cent $=\alpha(\mathrm{D} . \mathrm{M} .)^{-\beta}$ allowed determination of the level of nitrogen nutrition of each species. No positive effects of the presence of lucerne on the level of cocksfoot nitrogen nutrition were shown. On the contrary, lucerne appeared very competitive for mineral nitrogen, especially when its rate of growth was maximum, during summer regrowth. This species had the higher rate of regrowth and used most of the nitrogen applied at a given time. Nitrogen application to mixed stands amplified the dominance of one species over the other. The consequences of the nitrogen fertilization of lucerne-cocksfoot associations are discussed. In a pure stand of lucerne it appears that the very strong intraspecific competition could have a depressive effect on atmospheric utilization.

Additional key words : Dilution curves, $N$ content, Medicago sativa, Dactylis glomerata, competition.

\section{INTRODUCTION}

Dans l'article précédent (CRUZ \& LEMAIRE, 1986), nous avons étudié l'effet des compétitions inter et intraspécifiques, sur la croissance en matière sèche d'une luzerne et d'un dactyle associés. Pour interpréter plus complètement ces données, il est nécessaire de pouvoir estimer globalement l'effet de ces 2 types de compétition sur le niveau de nutrition azotée des 2 partenaires.

Dans une association graminée-légumineuse, coexistent les différents modes de nutrition azotée : assimilation de l'azote minéral chez la graminée et fixation symbiotique plus assimilation chez la légumineuse. 
Malgré l'idée généralement acceptée que lés graminées sont plus compétitives pour l'utilisation de l'azote minéral du sol, VaLLIS (1978) remarque que, dans certains cas, les légumineuses peuvent devenir elles aussi très agressives dans cette compétition (LORENTZ et al., 1961 ; MAC LEOD \& CARSON, 1965 ; REHM et al., 1975). Dans les associations où la composante légumineuse est représentée par la luzerne, des résultats contradictoires ont été obtenus (JACQUARD, 1977).

L'effet global bénéfique de la présence d'une légumineuse sur la nutrition azotée de la graminée a été signalé par de nombreux auteurs (VAN RIPER, 1964 ; BIRCH \& Dougall, 1967 ; ChARLES, 1982). Ces auteurs se basent sur des augmentations de teneurs en azote de la graminée associée par rapport à la culture pure pour en déduire l'existence d'éventuels transferts de l'azote fixé par la légumineuse vers la graminée. Cette notion de transfert d'azote est contestée par KILCHER et al. (1966) qui ont travaillé sur une association luzerne-graminée de même que par CHALAMET et al. (1983) et LEDGARD et al. (1985) qui n'ont pas pu mettre ce transfert en évidence par l'utilisation de l'azote marqué $\left(\mathrm{N}^{15}\right)$ sur diverses associations entre graminées et légumineuses.

L'objectif de ce travail est de pouvoir apporter des éléments de réponse aux questions suivantes :

- la présence de la luzerne améliore-t-elle la nutrition azotée du dactyle?

- la présence du dactyle modifie-t-elle la nutrition azotée de la luzerne ?

- quel est l'effet d'un apport d'azote minéral sur le niveau de nutrition azotée des 2 partenaires associés?

Les éléments de réponse à ces 3 questions seront apportés grâce à une méthode d'analyse permettant de caractériser globalement le niveau de nutrition azotée des 2 espèces dans les diverses situations expérimentales : cultures pures et associées. Cette analyse ne permet cependant pas de faire la part entre les 2 modes de nutrition : assimilation de l'azote minéral du sol et fixation symbiotique.

\section{MATÉRIEL ET MÉTHODE}

\section{A. Méthode d'estimation du niveau de nutrition azo- tée d'un couvert végétal}

\section{Rappel de la méthode des "courbes de dilution"}

Cette méthode a été développée à partir des études de SaleTte \& Lemaire (1981) qui ont montré que, sur des peuplements de graminées pures, on pouvait relier la cinétique de diminution de la teneur en azote total des parties aériennes au cours d'une repousse à la cinétique de croissance en matière sèche par une relation du type :

$$
\text { N p. } 100=\alpha(\mathrm{MS})^{-\beta}
$$

où $\alpha$ représente la teneur en azote des parties aériennes lorsque la production de matière sèche est de $1 \mathrm{t}$. ha ${ }^{-1}$ et $\beta$ représente un coefficient de dilution de l'azote.

Dans la figure 1, nous avons représenté schématiquement les courbes correspondant à plusieurs

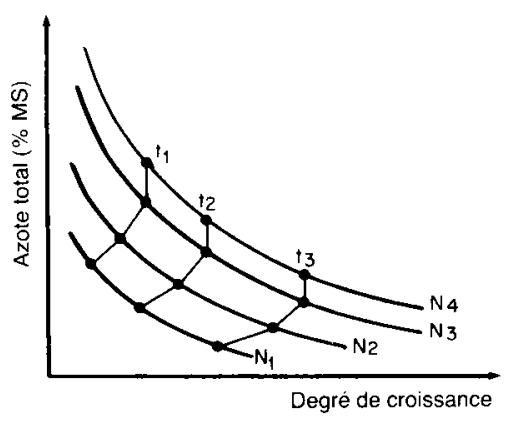

Figure 1

Schéma des courbes de dilution de l'azote dans la biomasse aérienne; scheme of the dilution curves of nitrogen in aerial biomass.

$\% N=\propto(M S)^{-\beta}$

$N_{1}, N_{2}, N_{3}, N_{4}=$ niveaux d'offre d'azote par le sol; level of nitrogen availability.

$t_{1}, t_{2}, t_{3}=$ temps de repousse; time of regrowth.

niveaux de nutrition azotée, c'est-à-dire à différentes offres d'azote par le milieu $\left(\mathrm{N}_{1}, \mathrm{~N}_{2}, \mathrm{~N}_{3}\right.$ et $\left.\mathrm{N}_{4}\right)$. L'accroissement de cette offre se traduit par une augmentation simultanée de la croissance et de la teneur en azote pour une date donnée. Au-delà du niveau $\mathrm{N}_{3}$, il peut y avoir un enrichissement en azote dans la plante sans augmentation de croissance. Cela indique le niveau à partir duquel les conditions de nutrition azotée sont non limitantes de la production de matière sèche. Les études plus précises de LemaIRE \& SALETTE (1984a et $b$ ) ont montré que les courbes correspondant au niveau de nutrition azotée non limitant pour la croissance en matière sèche étaient relativement identiques entre lieux, entre années et pour différents génotypes de graminées. Ainsi, on peut utiliser ces courbes comme références pour un diagnostic du niveau réel de nutrition azotée d'un couvert végétal en cours de croissance : la distance d'un point donné (N p. 100, MS) à la courbe de référence représente alors une indication du déficit de nutrition azotée.

Dans une étude précédente (LEMAIRE et al., 1985), nous avons montré que cette relation pouvait globalement s'appliquer à un peuplement de luzerne pure permettant ainsi de formuler un diagnostic global sur son niveau de nutrition azotée.

La figure 2 explique le mode d'utilisation des courbes de dilution pour l'interprétation des différences de teneur en azote. Ces différences peuvent avoir 2 causes possibles :

- soit une différence réelle de nutrition azotée (points a et $b$ ), auquel cas les 2 points se trouvent sur des courbes de dilution différentes,

- soit une différence de croissance en matièrè sèche non consécutive à une différence de nutrition azotée (points $\mathrm{c}$ et $\mathrm{b}$ ), auquel cas les 2 points sont situés sur la même courbe de dilution.

\section{Application aux cultures associées}

L'utilisation de la méthode des courbes de dilution dans le cas des cultures associées pose le problème de l'unité de mesure de la biomasse des 2 partenaires.

Le dispositif expérimental a été décrit précédemment (CRUZ \& LEMAIRE, 1986). Il s'agit de cultures associées en lignes alternées; la biomasse de chaque espèce peut donc être exprimée en $\mathrm{g} / \mathrm{m}$ linéaire. 


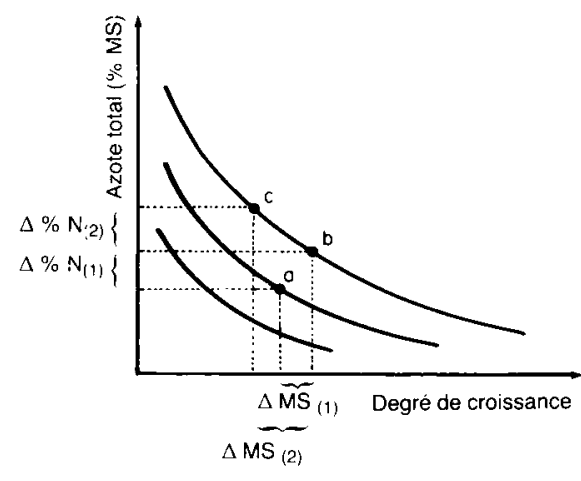

Figure 2

Différences des teneurs en azote dans la biomasse aérienne dues à :

Differences in nitrogen content in the aerial biomass due to :

(I) changement du niveau de nutrition azotée $:(b-a)$; a change in the level of nitrogen nutrition;

(2) dífférents degrés de croissance (même niveau de nutrition azotée) : $(b-c)$; different degrees of growth (same level of nitrogen nutrition).

La comparaison des courbes de dilution de chacune des 2 espèces en association peut donc se faire avec celles obtenues sur les lignes correspondantes en cultures pures. Comme dans l'analyse des croissances en matière sèche (CRUZ \& LEMAIRE, 1986), une séparation entre les effets de la compétition intraspécifique et de la compétition interspécifique peut être réalisée en utilisant pour chaque espèce les 3 peuplements :

- culture associée en lignes alternées $(20 \mathrm{~cm})$ : A,

- culture pure à écartement normal $(20 \mathrm{~cm}): \mathrm{E}$,

- culture pure à double écartement $(40 \mathrm{~cm}): 2 \mathrm{E}$.

Ainsi, la comparaison des différentes courbes de dilution obtenues dans les divers types de peuplement permet de mettre en évidence l'effet direct de la compétition sur le niveau de nutrition azotée en éliminant l'effet indirect sur les teneurs en azote consécutif à une modification de la cinétique de croissance.

Le protocole expérimental et la conduite des 2 expérimentations ont été décrits dans l'article précédent (CRUZ \& Lemaire, 1986). La détermination des teneurs en azote a été réalisée pour chaque traitement sur chacun des blocs. Les courbes de dilution ont été construites à partir des moyennes des teneurs en azote et des matières sèches récoltées. Compte tenu du faible nombre de points (de 5 à 8 ) pour construire chacune des courbes, nous n'avons pas jugé utile de calculer les équations d'ajustement au modèle, $\mathrm{N}$ p. $100=\alpha(\mathrm{MS})^{-\beta}$, étant donné le faible nombre de degrés de liberté pour comparer les valeurs des coefficients $\alpha$ et $\beta$. Nous nous sommes contenté d'une simple comparaison visuelle des courbes, en ne prenant en compte que les différences les plus évidentes.

\section{RÉSULTATS ET DISCUSSIONS}

\section{A. Comparaison des prélèvements d'azote des cultu- res pures et des associations}

Le tableau 1 permet de comparer les quantités d'azote prélevées à chaque coupe par les peuplements purs et par les peuplements associés. L'association $0 \mathrm{~N}$
TABLEAU 1

Prélèvement d'azote ( $\left.\mathrm{kg} . \mathrm{ha}^{-1}\right)$ des cultures pures et des associations pour les différentes repousses.

Nitrogen uptake $\left(\mathrm{kg} . \mathrm{ha}^{-1}\right)$ by pure stands and mixtures for different regrowths.

\begin{tabular}{|c|c|c|c|c|c|c|}
\hline \multicolumn{2}{|c|}{ Repousses } & \multirow{2}{*}{$\frac{1^{\mathrm{re}}}{164}$} & \multirow{2}{*}{$\frac{2^{e}}{110}$} & \multirow{2}{*}{$\frac{3^{e}}{124}$} & \multirow{2}{*}{$\frac{4^{e}}{96}$} & \multirow{2}{*}{$\frac{\text { Total }}{494}$} \\
\hline Luz. E & $0 \mathrm{~N}$ & & & & & \\
\hline Dact. E & $\mathrm{N}$ & 144 & 102 & 124 & 113 & 483 \\
\hline \multicolumn{2}{|c|}{ Moyennes cultures pures } & 154 & 106 & 124 & 104 & 488 \\
\hline Association & $1 / 2 \mathrm{~N}$ & & & & & \\
\hline Luzerne & & 115 & 89 & 116 & 78 & 398 \\
\hline Dactyle & & 71 & 42 & 21 & 18 & 152 \\
\hline Total & & 186 & 131 & 137 & 96 & 550 \\
\hline Association & $0 \mathrm{~N}$ & & & & & \\
\hline Luzerne & & 129 & 72 & 90 & 70 & 361 \\
\hline Dactyle & & 52 & 28 & 17 & 18 & 115 \\
\hline Total & & 181 & 100 & 107 & 88 & 476 \\
\hline
\end{tabular}

prélève plus d'azote que chacune des cultures pures au printemps ( $1^{\text {re }}$ repousse), par contre elle en prélève moins lors des repousses suivantes. Un apport d'azote sur l'association permet de maintenir un niveau de prélèvement supérieur à celui des cultures pures pendant les 3 premières repousses.

On peut noter la très faible contribution du dactyle dans l'association $0 \mathrm{~N}$ en $3^{\mathrm{e}}$ et $4^{\mathrm{e}}$ repousses. Cette contribution n'est pas (ou peu) améliorée par un apport d'azote, contrairement à celle de la luzerne. Il semble donc qu'en période estivale ce soit la luzerne qui, dans l'association, bénéficie le plus de l'azote minéral apporté ; au printemps, au contraire, c'est la nutrition azotée du dactyle qui est favorisée.

La comparaison des courbes de dilution obtenues dans chacun des peuplements doit nous permettre d'analyser cette compétition pour l'azote entre les 2 espèces.

\section{B. Effets de la présence de la luzerne sur le niveau de nutrition azotée du dactyle}

La comparaison des courbes de dilution du dactyle associé (A) avec celles du dactyle en culture pure à double écartement (2E) nous permet d'estimer l'effet de la présence des lignes adjacentes de luzerne sur le niveau de nutrition azotée du dactyle.

La figure 3a représente l'évolution des teneurs en azote du dactyle en fonction du temps. On constate effectivement un enrichissement apparent en azote du dactyle associé par rapport au dactyle pur, pour les 2 niveaux d'apport d'azote $0 \mathrm{~N}$ et $1 / 2 \mathrm{~N}$. Des résultats similaires ont été obtenus par de nombreux auteurs et les ont amenés à conclure à un effet améliorateur de la luzerne sur la nutrition azotée du dactyle (CRAIG et al., 1981 ; ARNAUd \& NiQUeUX, 1982 ; BROADBENT $e t$ al., 1982).

L'examen des courbes de dilution de la figure $3 b$ montre qu'en fait, en l'absence d'apport d'azote, les augmentations de teneurs en azote constatées sur la figure 3 sont entièrement explicables par l'effet dépressif de la luzerne sur la croissance du dactyle et qu'il ne s'agit aucunement d'une amélioration de sa nutrition azotée. Avec un apport d'azote, on constate même que la présence de la luzerne diminue le niveau de la nutrition azotée du dactyle. Il semble donc que, 

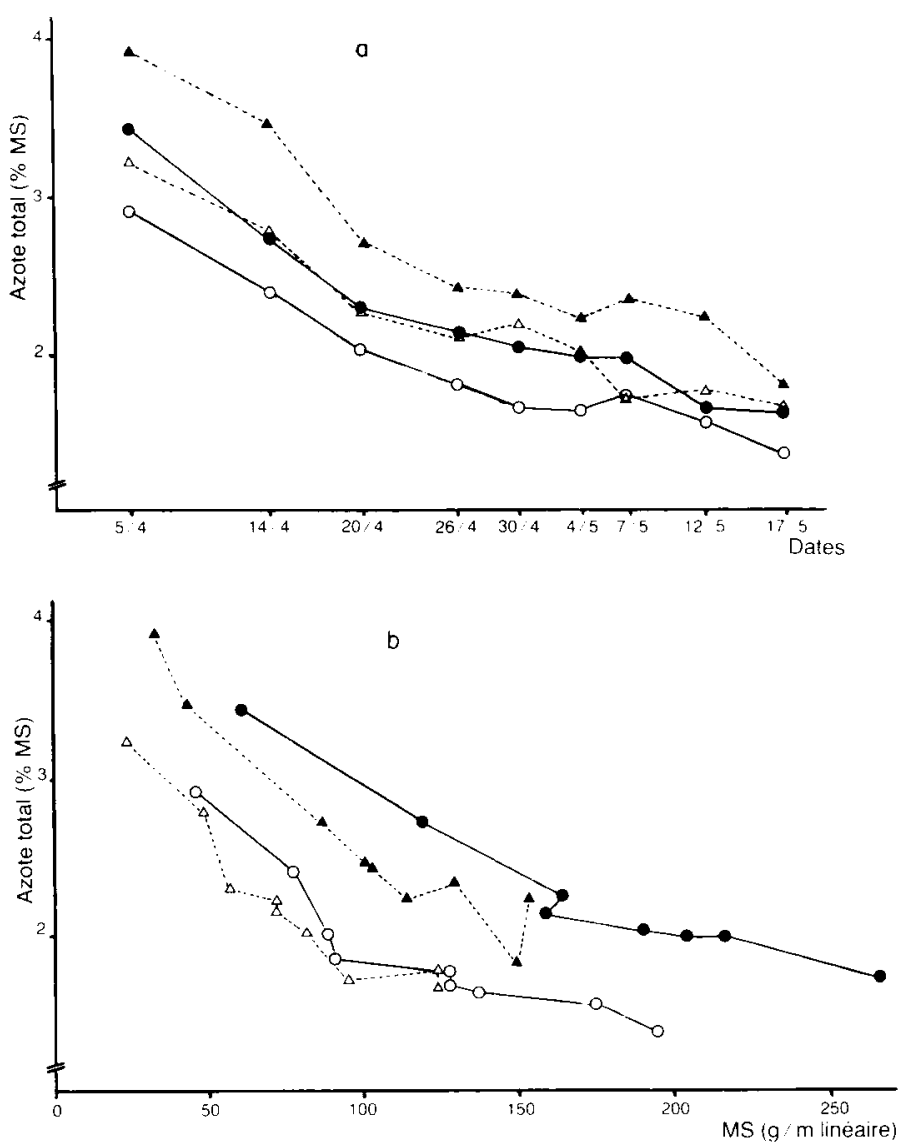

Figure 3

Evolution de la teneur en azote du dactyle au cours de la $I^{r e}$ repousse; change in the nitrogen content in cocksfoot during $I^{s t}$ regrowth:

a) en fonction du temps; with time;

b) en fonction de la matière sèche produite; in relation to dry matter accumulation;

- dactyle double écartement $+1 / 2 \mathrm{~N}$; cocksfoot double-spaced row $+1 / 2 N$

$O$ dactyle double écartement, oN; cocksfoot double-spaced row, ON:

$\Delta$ dactyle associé $+1 / 2 N$; in mixture $+1 / 2 N$;

$\triangle$ dactyle associé, $0 N$; in mixture, $0 N$.

lorsque la disponibilité en azote minéral du sol augmente, la luzerne devient compétitive vis-à-vis du dactyle pour son utilisation.

On pourrait penser que les transferts d'azote, nécessitant un recyclage par décomposition de la matière organique, ne puissent apparaître qu'avec un décalage d'une année. La figure 4 permet de montrer qu'au printemps 1983 nous ne mettons en évidence aucun effet bénéfique apparent. Ces résultats ne permettent pas de démontrer l'absence effective de transferts d'azote de la luzerne à la graminée, mais ils indiquent que, s'ils existent réellement, leur effet positif est très largement compensé par la capacité de concurrence de la luzerne pour l'utilisation de l'azote minéral disponible dans le sol.

\section{Effet de la présence du dactyle sur le niveau de nutrition azotée de la luzerne}

Les courbes de dilution pour la luzerne en culture pure (2E) et en association (A) sont représentées à la

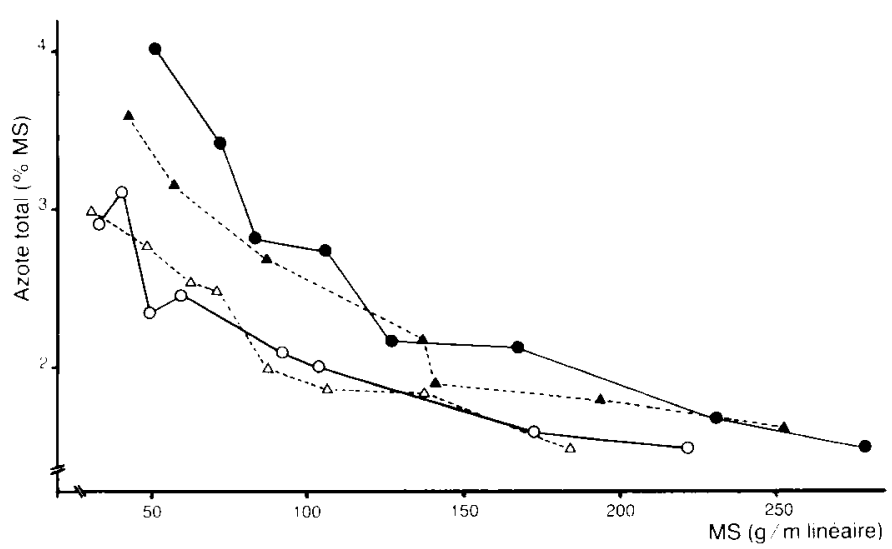

Figure 4

Courbes de dilution de l'azote dans la matière sèche produite par ligne de dactyle. Ire repousse 1983 (mêmes symboles que pour la figure 3).

Dilution curves of nitrogen in the dry matter accumulated by one row of cocksfoot during lst regrowth in 1983 (same symbols as in fig. 3).

figure 5. Nous avons privilégié l'analyse de la repousse de printemps et de la repousse estivale pour lesquelles nous obtenons des résultats contrastés.

Pour la repousse de printemps (fig. 5a), en l'absence d'apport d'azote, le dactyle exerce peu d'effet sur la nutrition azotée de la luzerne. On peut noter, cependant, un léger effet négatif en début de repousse, compensé par un effet positif par la suite. Après un apport d'azote, l'effet dépressif du dactyle en début de repousse est très marqué ; on retrouve cependant une tendance au rétablissement du niveau de nutrition azotée de la luzerne en fin de croissance. Ces résultats laissent supposer que la luzerne utiliserait une part non négligeable d'azote minéral en début de repousse, ce qui est attesté par l'amélioration de sa nutrition azotée en culture pure lorsque l'on passe du niveau $0 \mathrm{~N}$ au niveau $1 / 2 \mathrm{~N}$. Par contre, cette amélioration initiale se traduit par une détérioration du niveau de nutrition azotée, en fin de repousse, sans doute due à une inhibition partielle de sa capacité fixatrice par la présence abondante des nitrates (GROAT \& VANCE, 1980).

Le dactyle exerce une concurrence importante pour l'utilisation de l'azote minéral en début de repousse, ce qui a comme conséquence de diminuer l'effet négatif de la présence d'ions nitrate sur les capacités de fixation de la luzerne. Ceci permet d'expliquer les résultats sur les dynamiques de croissance présentés dans l'article précédent (CRUZ \& LEMAIRE, 1986) où nous avions constaté un effet positif de l'apport d'azote sur la croissance de la luzerne en début de repousse, compensé par un effet négatif assez net en fin de repousse.

Pour la repousse d'été (fig. 5b) en absence d'azote, le dactyle provoque une diminution très marquée du niveau de nutrition azotée de la luzerne, particulièrement en début de repousse. Par contre, avec un apport d'azote, l'effet dépressif du dactyle est beaucoup plus faible. Ceci correspond aux résultats présentés dans l'article précédent montrant l'absence de réponse de la croissance en matière sèche du dactyle associé à l'apport d'azote alors que la luzerne pure 

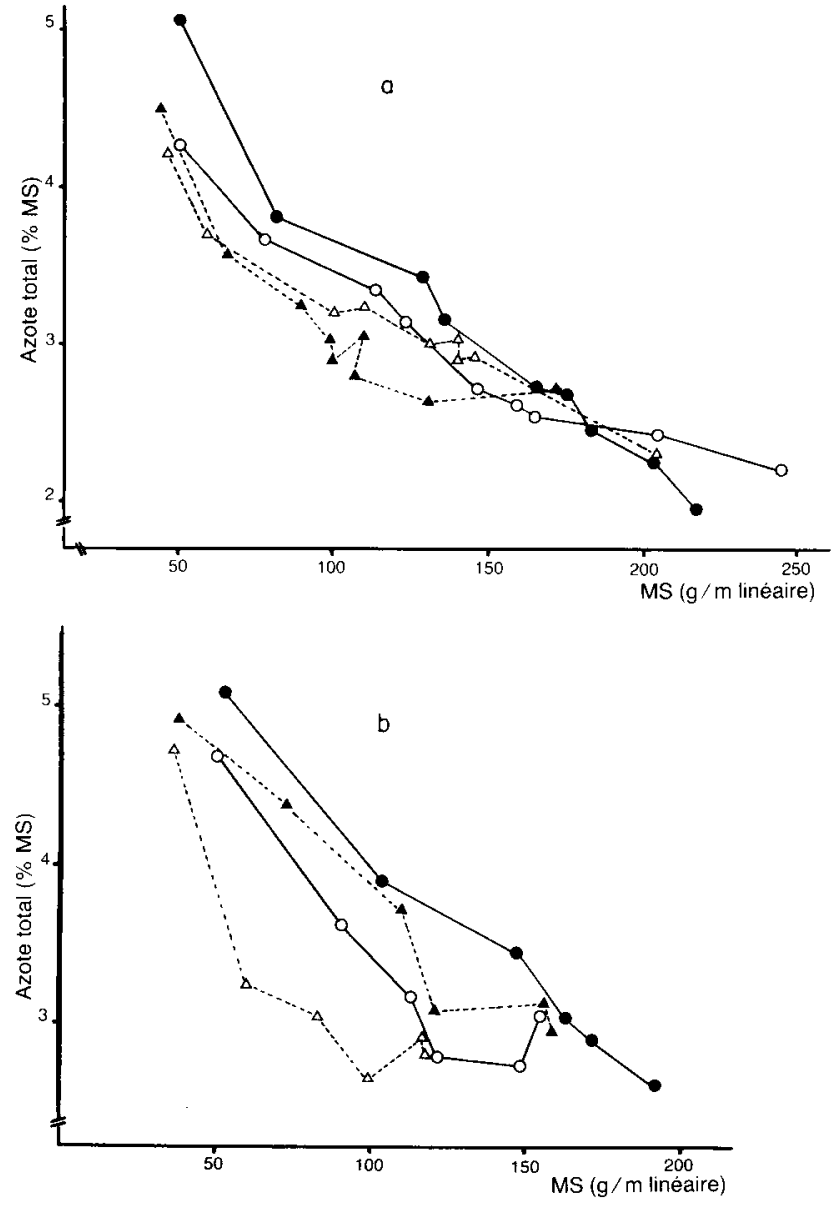

Figure 5

Courbes de dilution de l'azote dans la matière sèche produite par lignes de luzerne; dilution curves of nitrogen in the dry matter accumulated by one row of lucerne.

a) repousse de printemps; spring regrowth

b) repousse d'été, 1984 ; summer regrowth, 1984 ;

- luzerne double écartement, $1 / 2 N$; lucerne double-spaced row, $1 / 2 N$

luzerne double écartement, oN ; lucerne double-spaced row, ON ;

$\Delta$ luzerne associée, $1 / 2 N$; in mixture, $1 / 2 N$;

$\triangle$ luzerne associée, $O N$; in mixture, oN.

(2E) et la luzerne associée ont manifesté une réponse très importante.

\section{Analyse de l'effet d'un apport d'azote sur le niveau de nutrition azotée de la luzerne en culture pure et en association}

Les analyses précédentes nous ont montré qu'un apport d'azote minéral améliorait toujours le niveau de nutrition azotée de la luzerne en culture pure à double écartement. En culture associée, cette amélioration est surtout évidente pendant la croissance estivale lorsque la croissance du dactyle est suffisamment ralentie par la concurrence de la luzerne pour la lumière. Elle reste faible au printemps lorsque la croissance du dactyle est importante. Ceci suggère donc que la concurrence entre les 2 espèces pour l'utilisation de l'azote minéral est déterminée par le niveau relatif de leur capacité de croissance et donc de concurrence pour la lumière.

L'analyse plus complète des relations de compétition pour l'azote minéral entre les 2 espèces nécessite la prise en compte simultanée de la compétition intraspécifique.

L'expérimentation II nous permet d'en faire l'analyse pour la luzerne puisque nous disposons des courbes de dilution pour les 3 types de peuplement : luzerne pure $(\mathrm{E})$, luzerne pure $(2 \mathrm{E})$ et luzerne associée (A), avec 3 niveaux de nutrition azotée : $0 \mathrm{~N}$, $1 / 2 \mathrm{~N}$ et $\mathrm{N}$.

Les courbes de la figure 6 permettent de comparer l'effet d'un apport d'azote sur le niveau de nutrition azotée de la luzerne en culture pure (2E) et en association (A) : il s'agit de la compétition interspécifique.
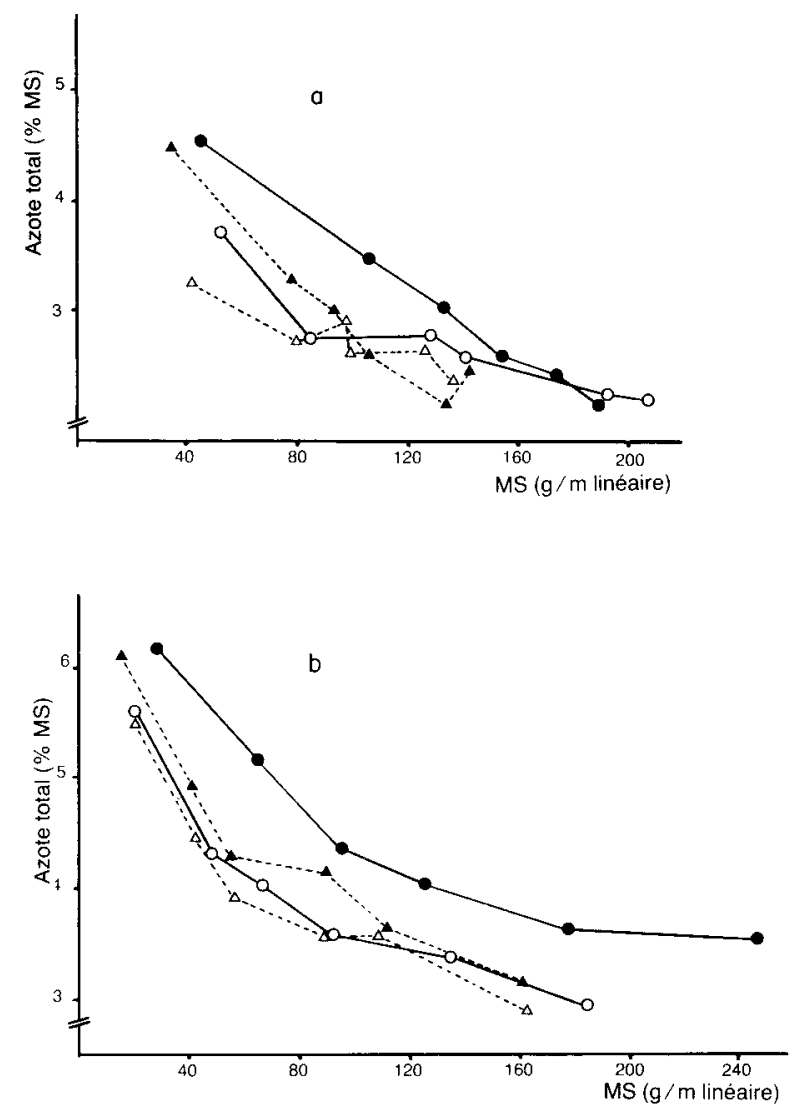

Figure 6

Courbes de dilution de l'azote dans la matière sèche produite par lignes de luzerne: dilution curves of nitrogen in the dry matter accumulated by one row of lucerne.

a) repousse de printemps; spring regrowth;

b) repousse d'été, 1982 ; summer regrowth, 1982 ;

- luzerne double écartement, $N$; lucerne double-spaced row, $N$;

O luzerne double écartement, oN; lucerne double-spaced row, oN,

- luzerne associée, $N$; in mixture, $N$;

$\triangle$ luzerne associée, $0 N$; in mixture, $0 N$.

Au printemps, nous confirmons les résultats obtenus lors de l'expérimentation I à savoir que, dans les 2 situations, l'apport d'azote minéral améliore la nutrition azotée en début de repousse, mais que cet effet est compensé en fin de repousse par une diminution (dilution plus rapide de l'azote). En été, on observe comme dans l'expérimentation I, une forte augmentation du niveau de nutrition azotée de la luzerne pure (2E) qui se prolonge pendant toute la durée de la repousse alors que cette augmentation reste très faible pour la luzerne associée. Ceci peut expliquer l'absence de réponse de la croissance de la luzerne associée comparativement à ce qui avait été 
mis en évidence dans l'expérimentation I (CRUZ \& LEMAIRE, 1986).

Les courbes de la figure 7 permettent de comparer l'effet d'un apport d'azote sur le niveau de nutrition azotée de la luzerne en culture pure (E) et en culture associée, c'est-à-dire lorsque l'on substitue une ligne sur 2 de luzerne par du dactyle : il s'agit d'un bilan entre la compétition interspécifique et la compétition intraspécifique.

$\mathrm{Au}$ printemps, on observe, en début de croissance, une nette augmentation du niveau de nutrition azotée de la luzerne à écartement normal $(E)$ en réponse à l'apport d'azote, ainsi que nous l'avons déjà signalé pour la luzerne à double écartement (2E). Néanmoins, la dilution de l'azote pour les lignes à écartement normal est très rapide et les courbes se retrouvent très vite au-dessous de celles de la luzerne associée. Ceci nous indique que, vis-à-vis de la nutrition azotée d'une ligne de luzerne, la concurrence du dactyle est plus faible que la propre concurrence de la luzerne, phénomène constaté pour tous les niveaux de fertilisation. Ce résultat peut paraître, a priori, assez surprenant, toutefois il pourrait être expliqué par 2 hypothèses non exclusives l'une de l'autre :

- la luzerne a pu avoir, en début de croissance, une capacité à prélever l'azote minéral plus importante que le dactyle. Rappelons la dépendance de la
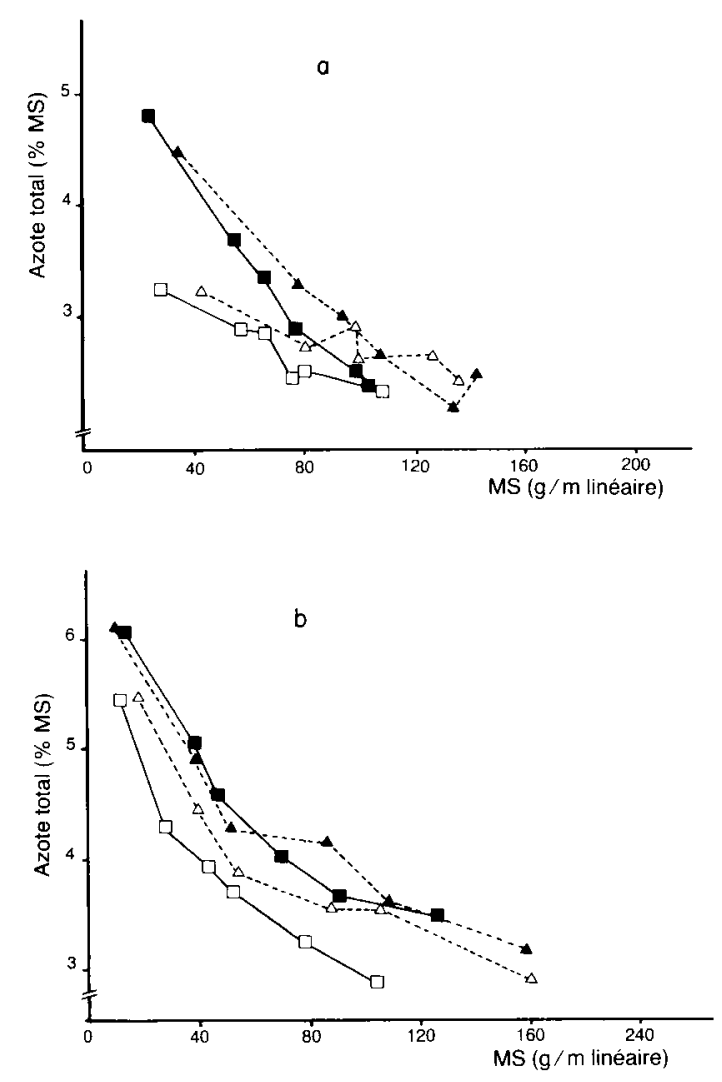

Figure 7

Courbes de dilution de l'azote dans la matière sèche produite par lignes de luzerne; dilution curves of nitrogen in the dry matter accumulated by one row of lucerne.

a) repousse de printemps; spring regrowth ;

b) repousse d'été, 1984 ; summer regrowth, 1984 ;

- luzerne écartement normal, $N$; lucerne single-spaced row, $N$; luzerne écartement normal, oN ; lucerne single-spaced row, oN;

$\Delta$ luzerne associée, $N$; in mixture, $N$;

$\triangle$ luzerne associée, oN ; in mixture, oN. luzerne vis-à-vis de la voie assimilatrice à ce stade (FISHBECK \& PHILlIPS, 1981 ; WERY, 1983) ;

- à cette $1^{\text {re }}$ explication peut s'en ajouter une autre : la concurrence pour la lumière entre lignes de luzerne à écartement normal serait suffisamment importante pour diminuer sa capacité de fixation d'azote par rapport à celle d'une ligne de luzerne en association avec le dactyle. L'effet d'un ombrage entre lignes de luzerne à écartement normal (E) réduit la disponibilité en lumière, donc en assimilats carbonés au niveau de chaque ligne. Il est bien connu que la disponibilité en carbone dans les nodules est un des principaux facteurs limitants pour la fixation d'azote (MURPHY, 1981 ; SCHRAMM, 1982).

Seules, bien entendu, des mesures directes de l'assimilation et de la fixation pourraient nous permettre d'apporter une explication définitive à ce problème.

En été, il existe un effet de l'apport d'azote sur la nutrition azotée de la luzerne pure à écartement normal qui ne se retrouve qu'à un degré moindre sur la luzerne associée. On observe que la dilution de l'azote au cours de ce cycle est moins rapide, notamment pour le traitement $\mathbf{N}$ comme nous l'avons vu pour le peuplement pur à double écartement à cette même période (fig. 6). Pour le niveau $0 \mathrm{~N}$, on vérifie que la courbe de dilution de la ligne de luzerne pure est inférieure à celle de la luzerne associée, tout au long de la période de repousse. Ceci confirme l'importante compétition intraspécifique qui jouerait sur la fixation. A la différence du $1^{\text {er }}$ cycle, on observe que cet effet dépressif disparaît lorsque l'on apporte de l'azote. Ceci pourrait indiquer que la ligne de luzerne à écartement normal compense sa plus faible fixation par l'utilisation de $\mathrm{NO}_{3}^{-}$. Ce phénomène de compensation n'a pas été observé au printemps. A cette période, la luzerne à écartement normal a été incapable de répondre à l'azote comme l'ont montré les courbes de croissance de l'article précédent (CRUZ \& LEMAIRE, 1986).

Le potentiel de croissance plus faible de la luzerne $(E, N)$, au printemps, peut expliquer cette absence de réponse. $\mathrm{Au} 1^{\text {er }}$ cycle, sa vitesse moyenne de croissance est de $99 \mathrm{~kg} \mathrm{MS} / \mathrm{ha} / \mathrm{j}$ tandis qu'en été elle est de $192 \mathrm{~kg} \mathrm{MS} / \mathrm{ha} / \mathrm{j}$. Ces différences de croissance engendrent des différences analogues dans les besoins instantanés en azote. Dans ces conditions, la plus forte demande en azote de la luzerne en été ne pourrait plus être satisfaite par la seule fixation.

\section{CONCLUSION}

L'analyse des différentes courbes de dilution présentée dans ce $2^{\mathrm{e}}$ article permet d'apporter des réponses, au moins partielles, aux questions que nous avions posées en introduction.

L'effet améliorateur de la luzerne sur la nutrition azotée du dactyle n'apparaît pas et il semble même qu'il existe une forte concurrence entre les 2 espèces pour l'utilisation de l'azote minéral apporté. Ces résultats mettent en évidence les erreurs d'interprétation qui peuvent être faites lorsque l'on se contente de caractériser le niveau de nutrition azotée d'un peuplement végétal par sa teneur en azote sans tenir compte de son degré de croissance.

L'effet du dactyle sur la nutrition azotée de la 
luzerne apparaît plus complexe. En début de croissance et notamment au printemps, il concurrence la luzerne pour l'utilisation de l'azote minéral du sol mais ceci peut être compensé par une meilleure fixation de la légumineuse en fin de croissance. Des études cinétiques, permettant de séparer les 2 voies de nutrition azotée, seraient nécessaires pour analyser plus précisément ce phénomène. Cependant la concurrence exercée par le dactyle sur la nutrition azotée de la luzerne reste toujours plus faible que l'effet dépressif de la luzerne vis-à-vis d'elle-même.

L'effet d'un apport d'azote sur le niveau de nutrition azotée des 2 partenaires de l'association apparaît être en premier lieu dépendant de leur capacité respective de croissance. Nous confirmons ainsi parfaitement les conclusions qui ont été émises dans l'article précédent (CRUZ \& LEMAIRE, 1986) : la fixation ne pourrait suffire à assurer les très fortes demandes en azote de la luzerne dans des conditions de croissance très rapides. Pour pouvoir être acceptée, cette hypothèse devra, bien entendu, être confrontée à des mesures réelles des capacités fixatrices de la luzerne.

Certains des résultats présentés ici apportent des éléments de réflexion sur l'utilisation de l'association graminée-légumineuse comme culture fourragère. On a montré qu'en absence d'apport d'azote, la nutrition azotée d'une espèce n'est que peu modifiée par la présence du partenaire. Cependant, dans les peuplements associés ayant reçu une fumure azotée, des concurrences importantes entre les 2 espèces sont mises en évidence. Il s'ensuit que, dans ces situations, l'azote amplifie la compétition pour la lumière entre les 2 espèces en augmentant préférentiellement la croissance de l'espèce dominante. Ainsi la survie de l'espèce dominée dépendra en fait de sa propre capacité de croissance. Si celle-ci reste malgré tout importante en valeur absolue, l'espèce dominée pourra se trouver en situation d'épuisement important de ses réserves carbonées faute d'un accès suffisant à la lumière, ce qui peut aboutir à son élimination. Si, par contre, la capacité de croissance de l'espèce dominée reste faible à cette période, le niveau des réserves peut rester suffisant pour assurer la repousse après la coupe et la survie des individus dans le peuplement se trouvera moins affectée par la compétition de l'autre espèce. La $1^{\text {re }}$ de ces 2 situations extrêmes peut être obtenue en utilisant des variétés synchrones (coïncidence des périodes à forte capacité de croissance des 2 espèces). La $2^{\mathrm{e}}$ situation est obtenue avec des variétés asynchrones (décalage des maximums de croissance).

Dans un système de production intensive, où l'utilisation de la fertilisation et de l'irrigation accentue la compétition, il pourrait être avantageux d'utiliser l'association d'espèces asynchrones. Ceci permettrait d'améliorer la pérennité du peuplement mixte et d'augmenter sa production totale annuelle par l'alternance dans le temps des cycles de production des partenaires (RoTILI et al., 1983).

Dans le cas où l'intensification de la production est moindre, l'utilisation d'espèces ou cultivars ayant les mêmes rythmes de croissance aurait des conséquences moins négatives. Ceci correspond aux associations non fertilisées ou à des conditions de déficit hydrique plus ou moins marqué.

Reçu le 29 novembre 1985. Accepté le 18 avril 1986.

\section{RÉFÉRENCES BIBLIOGRAPHIQUES}

Arnaud R., Niqueux M., 1982. Possibilités des associations du trèfle violet avec différentes graminées en moyenne montagne (BourgLastic, 1978-1982). Fourrages, 89, 3-28.

Birch H. F., Dougall H. W., 1967. Effect of a legume on soil nitrogen mineralization and percentage nitrogen in grasses. Plant Soil, 17 (2), 292-296.

Broadbent F. E., Nakashima T., Chang G. Y., 1982. Estimation of nitrogen fixation by isotope dilution in field and greenhouse experiments. Agron. J., 74 (4), 625-628.

Chalamet A., Audergon J. M., Maitre J. P., 1983. Concurrence entre une graminée (Lolium multiflorum Lamk.) et une légumineuse (Trifolium pratense L.) : utilisation du ${ }^{15} \mathrm{~N}$ pour l'étude de la nutrition azotée. Acta æcol. Oecol. Plant., 4 (18), 125-133.

Charles J. P., 1982. Utilisation en Suisse du trèfle violet et de la luzerne avec des graminées. Fourrages, 90, 39-56.

Craig L., Wiebold W. J., Mac Intosh M. S., 1981. Nitrogen fixation rates of alfalfa or red clover grown in mixture with grasses. Agron. J., 73 (6), 996-998.

Cruz P., Lemaire G., 1986. Analyse des relations de compétition dans une association de luzerne (Medicago sativa L.) et de dactyle (Dactylis glomerata L.). I. Effets sur les dynamiques de croissance en matière sèche. Agronomie, 6 (8).

Fishbeck K. A., Phillips P. A., 1981. Combined nitrogen and vegetative regrowth of symbiotically-grown alfalfa. Agron. J., 73 (6), 975-978.
Groat R. G., Vance C. P., 1981. Harvest and nitrogen fertiliser effects on alfalfa root nodule enzymes of ammonia assimilation. Agric. Rev. and Manuals, U.S. Dep. Agric., 19, 64-65.

Jacquard P., 1977. Relations entre espèces dans les associations graminées-légumineuses. Sel. $f r$., 24, 3-28.

Kilcher M. R., Clark K. W., Heinrichs D. H., 1966. Dryland grassalfalfa mixture yields and influence of associats on one another. Can. J. Plant Sci., 46 (3), 279-284.

Ledgard S. F., Simpson J. R., Freney J. R., Bergersen F. J., 1985. Field evaluation of $\mathrm{N}^{15}$ techniques for estimating nitrogen fixation in legume-grass associations. Aust. J. agric. Res., 36, 247-258.

Lemaire G., Salette J., 1984a. Relations entre dynamique de croissance et dynamique de prélèvement d'azote pour un peuplement de graminées fourragères. I. Etude de l'effet du milieu. Agronomie, 4 (5), 423-430.

Lemaire G., Salette J., 1984b. Relation entre dynamique de croissance et dynamique de prélèvement d'azote pour un peuplement de graminées fourragères. II. Etude de la variabilité entre génotypes. Agronomie, 4 (5), 431-436.

Lemaire G., Cruz P., Gosse G., Chartier M., 1985. Etudes des relations entre la dynamique de prélèvement d'azote et la dynamique de croissance en matière sèche d'un peuplement de luzerne (Medicago sativa L.). Agronomie, 5 (8), 685-692. 
Lorentz R. J., Carlson C. W., Rogler G. A., Holman H., 1961 Bromegrass and bromegrass-alfalfa yields as influenced by moisture level, fertilizer rates and harvest frequency. Agron. J. 53 (1), 49-52.

Mac Leod L. B., Carson R. B., 1965. Effect of source and rate of $N$ and rate of $\mathrm{K}$ on the yield and chemical composition of alfalfa and orchardgrass. Can. J. Plant Sci., 45, 557-569.

Murphy P. M., 1981. Photosynthate supply and nitrogen fixation in forage legumes. Proc. I4th int. Grassl. Congr, Lexington, Kentucky, p. 147 .

Rehm G. W., Nichols J. T., Sorensen R. C., Moline W. J., 1975. Yield and botanical composition of an irrigated grass-legume pasture as influenced by fertilization. Agron. J., 67 (1), 64-68.

Rotili P., Zannone L., Scotti C., Paoletti R., Gnocchi G., Romani M., 1983. Interferenza interspecifica in piante foraggere. II. Comportamento delle associazioni di erba medica con graminacee in strutture prative construite a linee alterne et binate. Ann. Ist. sper. Colt. Foraggere, 6, 71-114.
Salette J., Lemaire G., 1981. Note sur la variation de la teneur en azote des graminées fourragères pendant leur croissance : formulation d'une loi de dilution. C. R. Acad. Sci., Paris, Ser. 3, 875-878.

Schramm R. W., 1982. Carbon assimilation and flux connected with nitrogen fixation in legumes. Isr. J. Bot., 31, 131-139.

Vallis I., 1978. Nitrogen relationships in grass/legume mixture, 190 201. In: J. R. Wilson « Plant relations in pastures». CSIRO, Camberra, 439 p.

Van Riper G. E., 1964. Influence of soil moisture on the herbage of two legumes and three grasses as related to dry matter yields, crude protein and botanical composition. Agron. J., 56 (1), 45-50.

Wery J., 1983. Contribution à l'étude de la nutrition azotée d'une légumineuse fourragère (Medicago sativa $L$.) et de légumineuses à graines. Thèse Doct. Ing. Ec. nat. sup. agron., Montpellier, 239 p. 